\title{
EFEITO DO ESTRESSE SALINO E DA PROLINA EXÓGENA EM CALOS DE MILHO ${ }^{1}$
}

\author{
TEREZINHA RANGEL CAMARA ${ }^{2}$, LILIA WILLADINO ${ }^{3}$, JOSEP MARIA TORNÉ ${ }^{4}$, \\ ALBERT MANICK ${ }^{5}$ E MARIA ASUNCIÓN SANTOS ${ }^{4}$
}

Universidade Federal Rural de Pernambuco - Departamento de Química

\begin{abstract}
RESUMO - $O$ presente trabalho teve por objetivo avaliar o efeito de diferentes concentrações de $\mathrm{NaCl}(0$, 68, 137 e $205 \mathrm{mM}$ ) sobre o cultivo in vitro de calos de dois genótipos (W64Ao2 e Arizona 8601) de milho (Zea mays L.), em meio de cultura N6, suplementado ou não com a adição de $6,0 \mathrm{mM}$ de prolina. Os tratamentos consistiram da combinação dos quatro níveis de $\mathrm{NaCl}$ com os dois níveis de prolina, num fatorial $4 \times 2$. Os tratamentos foram mantidos durante 60 dias, com subcultivos a cada 20 dias. A taxa de crescimento dos calos e o conteúdo endógeno de putrescina, espermina, espermidina, prolina e outros aminoácidos livres foram avaliados no final do período experimental. A adição de prolina exógena favoreceu o crescimento dos calos no tratamento-controle e minorou os efeitos deletérios do estresse salino em ambos os genótipos, no nível de sal mais elevado $(250 \mathrm{mM}$ de $\mathrm{NaCl})$. A taxa de crescimento dos calos de W64Ao2 foi superior àquela dos calos de Arizona 8601, possivelmente por causa, em parte, do maior acúmulo de prolina endógena e da manutenção ativa do metabolismo das poliaminas naqueles calos.
\end{abstract}

TERMOS ADICIONAIS PARA INDEXAÇÃO: Aminoácidos, poliaminas, salinidade, Zea mays.

\section{EFFECT OF SALINE STRESS AND EXOGENOUS PROLINE IN MAIZE CALLUS}

\begin{abstract}
This work aimed at evaluating the effect of different $\mathrm{NaCl}$ concentrations $(0,68,137$ and $205 \mathrm{mM}$ ) in two maize (Zea mays L.) genotypes (W64Ao2 and Arizona 8601) callus cultures in N6 medium, supplemented or not with $6.0 \mathrm{mM}$ proline. Treatments consisted of the combinations of the four $\mathrm{NaCl}$ levels with the two-proline levels under $4 \times 2$ factorial. Treatments were maintained for 60 days with subcultures at 20 days each. Relative growth rate, endogenous free putrescine, spermine, spermidine, proline and others amino acids were evaluated at the end of the experimental period. Proline addition improved callus growth in both genotypes in the control treatment and reduced deleterious effects in the highest salt level $(250 \mathrm{mM} \mathrm{NaCl})$. W64Ao2 callus growth rate was higher than in the Arizona 8601 probably due, in part, to the higher proline accumulation and the maintenance of polyamine metabolism in those calluses.
\end{abstract}

ADDITIONAL INDEX TERMS: Aminoacids, poliamines, salinity, Zea mays.

1. Recebido em 21/2/2000 - Aceito em 6/7/2000

2. Agrônoma, Dra., Profa. Adjunto/UFRPE, Pesquisadora CNPq. e-mail tcamara@novaera.com.br

3. Bióloga, Dra., Profa. Adjunto/UFRPE, Pesquisadora do CNPq.

4. Biólogo, Dr., Centro de Investigación y Desarrollo - CSIC, Barcelona, Espanha.

5. Estatístico, Dr., Centro de Investigación y Desarrollo - CSIC, Barcelona, Espanha. 


\section{INTRODUÇÃO}

O impacto da salinidade do solo sobre a produtividade agrícola é um sério problema em regiões áridas e semi-áridas, que dependem da irrigação para garantir um adequado suprimento de água para as culturas (Tal, 1984). Dentre os mecanismos de tolerância ao estresse salino, destaca-se a osmorregulação, a qual inclui um aumento líquido na concentração de solutos nas células. $\mathrm{O}$ aumento na concentração de solutos em plantas submetidas a estresse desempenha um papel fundamental no equilíbrio osmótico e na proteção de enzimas em presença de elevadas concentrações de eletrólitos no citoplasma (Greenway e Munns, 1980).

Dentre os solutos orgânicos que se acumulam no citoplasma em resposta ao estresse, destacam-se a prolina (Kuznetsov e Shevyakova, 1997; Viégas et al., 1999), a putrescina (Basu e Gosh, 1991; Camara et al., 1998) e carboidratos solúveis (Garcia et al., 1997). Tem-se demonstrado uma correlação positiva entre a acumulação de prolina e a tolerância ao estresse salino, tanto em trabalhos realizados com cultivo in vitro (Jain et al., 1991; Camara et al., 1998), como naqueles envolvendo a planta inteira (Storey et al., 1993). Entretanto, a acumulação de prolina pode também ser interpretada como sintoma de danos causados na planta pelo estresse (Hasegawa et al., 1986; Das et al., 1990). Bellinger et al., (1991) sugerem que a acumulação de prolina não é um indicador de resistência e sim um indicador de tolerância adquirida, visto que diversos experimentos demonstram que células, calos e somaclones selecionados como tolerantes ao estresse apresentam uma maior acumulação de prolina do que os não adaptados.

O aumento da concentração de putrescina, como conseqüência do estresse salino, é uma resposta bastante generalizada em plantas (Shevyakova et al., 1985; Basu e Gosh, 1991). Por outro lado, a tolerância ao estresse salino parece estar associada não apenas à capacidade de acumular putrescina, mas em manter ativo o metabolismo das poliaminas, incluindo-se aí a síntese de espermina e espermidina (Tattini et al., 1993; Willadino et al., 1996).
Considerando a importância da acumulacão de solutos compatíveis, sobretudo a prolina, o presente trabalho teve como objetivo avaliar o efeito da adição de prolina ao meio de cultura sobre o crescimento de calos de milho submetidos ao estresse salino. Avaliou-se também o efeito da salinidade sobre a acumulação de putrescina, espermina, espermidina, prolina $\mathrm{e}$ alguns outros aminoácidos.

\section{MATERIAL E MÉTODOS}

Foram utilizados calos com 14 meses de idade, provenientes do cultivo de embriões imaturos de dois genótipos de milho: a linha pura W64Ao2, de origem chinesa; e o híbrido Arizona 8601, desenvolvido no Texas para cultivo em áreas salinas (Day, 1987).

Os calos foram obtidos e multiplicados em meio básico N6 (Chu et al., 1975), modificado com 6,0 mM de prolina (Claparols et al., 1993), sendo mantidos em sala de crescimento com fotoperíodo de 16 horas, fluxo de fótons fotossintético de 90-110 $\mu \mathrm{mol} \quad \mathrm{m}^{-2} \mathrm{~s}^{-1}$ e temperatura de $25 \pm 2^{\circ} \mathrm{C}$. Foram realizados três subcultivos, sendo um a cada 20 dias. Os tratamentos consistiram da adição de $0,68,137$ e $205 \mathrm{mM}$ de $\mathrm{NaCl}$ ao meio de cultivo, em combinação com 0 e $6 \mathrm{mM}$ de prolina, em um fatorial $4 \times 2$, inteiramente casualizado, com quatro repetições por tratamento. A taxa de crescimento dos calos (TCC) foi calculada a partir da massa fresca dos calos no início e final do experimento, de acordo com a equação: $\mathrm{TCC}=$ [(ln $\left.\mathrm{mf}_{\mathrm{f}}-\ln \mathrm{mf}_{\mathrm{i}}\right) /\left(\mathrm{t}_{\mathrm{f}}-\mathrm{t}_{\mathrm{i}}\right)$ x $\left.10^{3}\right]$ (Galiba et al.,1993), em que $\mathrm{mf}_{\mathrm{f}} \mathrm{e} \mathrm{mf}_{\mathrm{i}}$ correspondem à massa fresca final e inicial, e $t_{\mathrm{f}}$ e $t_{i}$ ao tempo final (60 dias) e inicial, respectivamente.

Sessenta dias após o início dos tratamentos, foram coletadas amostras para as análises bioquímicas. Essas amostras foram congeladas em nitrogênio líquido e mantidas à temperatura de $-80 \mathrm{oC}$ até a sua utilização.

As análises de poliaminas (livres + conjugadas) foram realizadas por cromatografia de camada delgada (TLC), segundo Tiburcio et al. (1985). Foi determinada a concentração de 
putrescina (Put), espermidina ( $\mathrm{Spd})$, espermina (Spm) e calculado o total de poliaminas (PAs), a partir do somatório da concentração de Put, de Spd e de Spm no tecido.

As concentrações endógenas de prolina (Pro), arginina (Arg), alanina (Ala), glutamina (Gln), glutamato (Glu), ácido $\gamma$-aminobutírico (GABA) e do total de aminoácidos nos calos foram determinadas por cromatografia líquida de alta resolução (HPLC), utilizando uma coluna Pico-Tag C-18 de 300 x 3,1 $\mathrm{mm}$. Para detecção dos aminoácidos, foi utilizado um detector de ultra-violeta a $254 \mathrm{~nm}$ (Pons et al., 1991). Problemas técnicos no preparo dos extratos de amostra dos calos de W64Ao2 tratados com $205 \mathrm{mM}$ de $\mathrm{NaCl}$ provocaram a perda do material, impossibilitando a realização das análises de aminoácidos nesses calos.

Os resultados obtidos foram submetidos à análise de variância e as médias foram comparadas pelo teste de Tukey, a 5\% de probabilidade, utilizando-se o Statgraph 5.0.

\section{RESULTADOS E DISCUSSÃO}

Em ambos os genótipos observou-se um decréscimo na taxa de crescimento dos calos em função do aumento dos níveis de salinidade no meio de cultura (Tabela 1). Por outro lado, a adição de prolina ao meio de cultura favoreceu 0 crescimento dos calos no tratamento sem $\mathrm{NaCl}$ e minorou o efeito deletério do estresse salino na dose de sal mais elevada $(205 \mathrm{mM})$ promovendo, simultaneamente, maior TCC e maior acúmulo de prolina livre (Tabela 2), em comparação com os calos tratados sem prolina exógena. $\mathrm{O}$ efeito benéfico da aplicação de prolina tem sido verificado em diversos tipos de estresse como o salino (Roy et al., 1993), o hídrico (Handa et al., 1986) e aquele induzido pelo frio (Songstad et al., 1990). A prolina ministrada exogenamente pode facilitar o ajuste osmótico e, conseqüentemente, favorecer a manutenção do crescimento sob condições de estresse salino (Hasegawa et al., 1986). Os calos de W64Ao2 tratados com prolina mantiveram uma taxa de crescimento positiva em todos os níveis de salinidade, enquanto que os de Arizona 8601 apresentaram taxa de crescimento negativa a partir do tratamento com $137 \mathrm{mM}$ de $\mathrm{NaCl}$. Em experimentos anteriores realizados com calos embriogênicos desses mesmos genótipos, submetidos a um menor tempo de cultivo in vitro, Willadino et al., (1996) obtiveram um maior crescimento e acúmulo de prolina livre dos calos de Arizona 8601 frente aos calos de W64Ao2 quando tratados com $\mathrm{NaCl}$. Deste modo, a exemplo do constatado também por outros pesquisadores (Evans, 1989; Bourgeais-Chalou e Guerrier, 1992) variações induzidas pelo tempo de cultivo in vitro podem alterar a resposta dos calos à salinidade.

A composição quantitativa de aminoácidos livres dos calos tratados com e sem prolina, foi afetada pela salinidade do meio (Tabelas 2 e 3). Os tratamentos com 137 e $205 \mathrm{mM}$ de $\mathrm{NaCl}$, em comparação com o tratamento sem sal, promoveram, de uma maneira geral, um acúmulo de Arg, Gln e Glu nos calos de Arizona 8601. No que se refere aos calos de W64Ao2 tratados com $137 \mathrm{mM}$ de $\mathrm{NaCl}$, observou-se um aumento na concentração de Arg, GABA, Ala, Glu e total de Aas, em relação aos calos mantidos sem $\mathrm{NaCl}$. A acumulação de Arg e seus precursores (Gln e Glu) foi observada também em células de Nicotiana sylvestris (Shevyakova et al., 1985) e em calos de milho (Willadino et al., 1996) submetidos ao estresse salino. Vários autores demonstraram que o estresse salino promove um incremento na atividade das enzimas glutamina sintetase (GS) (Roosens et al., 1998; Viégas e Silveira, 1999) e glutamato sintase (GOGAT) (Berteli et al., 1995) envolvidas na assimilação do íon amônio. O ciclo GS/GOGAT provavelmente desempenha um importante papel no suprimento de glutamato para a biossíntese de arginina e prolina em condições de estresse salino (Berteli et al., 1995). A elevada produção de $\mathrm{NH}_{3}-\mathrm{NH}_{4}^{+}$, durante o estresse abiótico, acompanhada de um incremento da via biossintética da Arg, é considerada um mecanismo de desintoxicação do íon amônio produzido (Slocum e Weinstein, 1990; Lovatt, 1990). A capacidade de incorporar esse amônio em condições de estresse, seja salino (Lovatt, 1990), osmótico (Flores e Galston, 1982) ou nutricional (Rabe e Lovatt, 1986), pode 
representar um mecanismo homeostático importante frente a condições de estresse em vegetais. $\mathrm{O}$ aumento na concentração de Pro livre em condições de estresse é amplamente documentado, tanto em estresse hídrico (Voetberg e Sharp, 1991; van Rensburg et al., 1993) como em estresse salino (Bourgeais-Chaillou e Guerrier, 1992; Cachorro et al., 1993). Em ausência de prolina exógena, o maior acúmulo de prolina livre nos calos de W64Ao2 submetidos ao estresse salino coincidiu com uma maior taxa de crescimento desses calos em comparação com os de Arizona 8601. A prolina parece fazer frente ao efeito inibidor do $\mathrm{NaCl}$ sobre o crescimento, contribuindo para uma maior adaptação das plantas e tecidos submetidos a condições adversas (Delauney e Verma, 1993). Entre as diversas funções atribuídas à prolina em tecidos vegetais submetidos a estresse destacam-se a osmorregulação, a manutenção do $\mathrm{pH}$ citoplasmático, a proteção contra a desnaturação de enzimas, o seqüestro de radicais livres, além de servir como reserva de carbono e nitrogênio e ser um dos produtos de desintoxicação do íon amônio (Bellinger et al., 1991; Alia et al., 1993; Fedina et al., 1994). A maior concentração de prolina nos calos tratados com $137 \mathrm{mM}$ de $\mathrm{NaCl}$ (Tabela 2) em relação ao mais alto nível de sal $(205 \mathrm{mM})$ nos calos de Arizona 8601 (Tabela 2) coincidiu com uma tendência inversa quanto ao conteúdo de PAs (Tabela 4). Esse mesmo comportamento foi observado nos tratamentos com $\mathrm{NaCl}$ e Pro exógena, para os dois genótipos avaliados. Ainda que a principal via biossintética da Pro seja por meio da ciclização do Glu, a Orn e a Arg (precursores das PAs) também podem ser metabolizadas a Pro (Thompson, 1980). Tanto em halófitas como em glicófitas (Kiryan e Shevyakova, 1984), Orn e Arg administradas exogenamente foram, em parte, desviadas para a síntese de Pro em condições de estresse salino. Esses resultados demonstram como a via metabólica das PAs e da Pro estão interligadas em condições de estresse salino, como demonstrado por outros autores (Shevyakova et al., 1985; Camara et al., 1998).

TABELA 1 - Taxa de crescimento dos calos (TCC) de dois genótipos de milho, em função dos tratamentos com (c) e sem (s) prolina e dos níveis de $\mathrm{NaCl}$.

Arizona 8601

W64Ao2

\begin{tabular}{|c|c|c|c|c|}
\hline \multirow{2}{*}{$\begin{array}{l}\mathrm{NaCl} \\
\mathrm{mM}\end{array}$} & \multirow[b]{2}{*}{ s/Pro } & \multirow[b]{2}{*}{ c/Pro } & \\
\hline & & & s/Pro & c/Pro \\
\hline 0 & $25,58 \mathrm{~b}$ & $46,43 a$ & $31,91 b$ & $39,30 \mathrm{a}$ \\
\hline 68 & $28,16 \mathrm{~b}$ & $27,17 b$ & $29,06 \mathrm{c}$ & $33,18 b$ \\
\hline 137 & $-5,03 c$ & $-6,38 \mathrm{~cd}$ & $11,06 \mathrm{~d}$ & $11,64 d$ \\
\hline 205 & $-17,01 \mathrm{e}$ & $-8,59 d$ & $-4,92 f$ & $3,21 \mathrm{e}$ \\
\hline
\end{tabular}

Os valores com a mesma letra, para cada genótipo, não apresentam diferença significativa pelo teste de Tukey ao nível de $5 \%$ de probabilidade. 
TABELA 2 - Concentração de aminoácidos totais (Aas), Prolina, Arginina e Ácido $\gamma$-aminobutírico (GABA) em calos de dois genótipos de milho, em função dos tratamentos com (c) e sem (s) prolina e dos níveis de $\mathrm{NaCl}$.

\begin{tabular}{|c|c|c|c|c|c|c|c|c|}
\hline \multirow{2}{*}{$\begin{array}{c}\mathrm{NaCl} \\
\mathrm{mM}\end{array}$} & \multicolumn{2}{|c|}{ Total de Aas } & \multicolumn{2}{|c|}{ Prolina } & \multicolumn{2}{|c|}{ Arginina } & \multicolumn{2}{|c|}{ GABA } \\
\hline & s/Pro & c/Pro & s/Pro & c/Pro & s/Pro & $\mathbf{c} / \mathbf{P r}$ & s/Pro & c/Pro \\
\hline \multicolumn{9}{|c|}{$\mu \mathrm{mol} \mathrm{kg} \mathrm{mf} \mathbf{~}^{-1}$} \\
\hline \multicolumn{9}{|c|}{ Arizona 8601} \\
\hline 0 & $34.656 \mathrm{~cd}$ & $22.955 \mathrm{e}$ & $731 \mathrm{f}$ & $6.664 \mathrm{c}$ & $314 \mathrm{c}$ & $101 \mathrm{e}$ & $8.040 \mathrm{~b}$ & $4.399 \mathrm{~cd}$ \\
\hline 68 & $16.206 \mathrm{f}$ & $31.698 \mathrm{~d}$ & $792 f$ & $8.993 b$ & $182 \mathrm{e}$ & $243 d$ & $3.301 \mathrm{~d}$ & $5.003 \mathrm{c}$ \\
\hline 137 & $34.318 \mathrm{~cd}$ & $40.467 b$ & $1.826 \mathrm{e}$ & $11.039 \mathrm{a}$ & $617 \mathrm{a}$ & $323 c$ & $7.047 \mathrm{~b}$ & $2.759 \mathrm{~d}$ \\
\hline 205 & $36.970 \mathrm{c}$ & $46.019 \mathrm{a}$ & $817 f$ & $4.858 \mathrm{~d}$ & $402 b$ & $615 a$ & $20.117 \mathrm{a}$ & $21.863 \mathrm{a}$ \\
\hline
\end{tabular}

W64Ao2

\begin{tabular}{|c|c|c|c|c|c|c|c|c|}
\hline 0 & $22.944 d$ & $46.406 \mathrm{~b}$ & $545 \mathrm{e}$ & $19.657 b$ & $0,0 \mathrm{e}$ & $0,0 \mathrm{e}$ & $1.985 \mathrm{c}$ & $2.620 \mathrm{~b}$ \\
\hline 68 & $25.442 d$ & $44.182 b$ & $991 \mathrm{e}$ & $21.715 a$ & $142 b$ & $67 \mathrm{c}$ & $2.240 \mathrm{c}$ & $2.071 \mathrm{c}$ \\
\hline 137 & $42.120 \mathrm{c}$ & $58.704 a$ & $1.743 d$ & $15.690 \mathrm{c}$ & $50 \mathrm{~d}$ & $201 \mathrm{a}$ & $4.512 \mathrm{a}$ & $4.197 \mathrm{a}$ \\
\hline 205 & - & - & - & - & - & - & - & - \\
\hline
\end{tabular}

Os valores com a mesma letra, para cada variável e genótipo, não apresentam diferença significativa, pelo teste de Tukey ao nível de 5\% de probabilidade.

Quanto ao efeito isolado da salinidade (sem prolina exógena), observou-se nos calos de W64Ao2 um acúmulo de PAs totais em todos os tratamentos com NaCl (Tabela 4). Os calos de Arizona 8601, entretanto, apresentaram, nas doses mais baixas de $\mathrm{NaCl}(68$ e 137 mM $\mathrm{NaCl}$ ), uma redução na concentração total de PAs em relação ao tratamento-controle. Quando submetidos ao tratamento com $205 \mathrm{mM}$ de $\mathrm{NaCl}$, registrou-se nesses calos um aumento na concentração total de PAs como reflexo da acumulação de Put e Spm. Por outro lado, o aumento na concentração total de PAs, nos calos de W64Ao2, refletiu o acúmulo de Put, bem como de Spd e Spm. Nesses calos, parece não haver ocorrido inibição da síntese das poliaminas com o aumento da salinidade. A capacidade de manter ativo o metabolismo das poliaminas em condições 
TABELA 3 - Concentração de Alanina, Glutamina e Glutamato em calos de dois genótipos de milho, em função do tratamento com (c) e sem (s) prolina exógena e dos níveis de $\mathrm{NaCl}$.

\begin{tabular}{|c|c|c|c|c|c|c|}
\hline \multirow{2}{*}{$\begin{array}{c}\mathrm{NaCl} \\
\mathrm{mM}\end{array}$} & \multicolumn{2}{|c|}{ Alanina } & \multicolumn{2}{|c|}{ Glutamina } & \multicolumn{2}{|c|}{ Glutamato } \\
\hline & s/Pro & c/Pro & s/Pro & c/Pro & s/Pro & c/Pro \\
\hline \multicolumn{7}{|c|}{$\mu \mathrm{mol}$ kg mf $\mathbf{f}^{-1}$} \\
\hline \multicolumn{7}{|c|}{ Arizona 8601} \\
\hline 0 & $18.747 \mathrm{a}$ & $7.748 \mathrm{~d}$ & $1.643 b$ & $857 \mathrm{e}$ & $1.111 \mathrm{e}$ & $373 f$ \\
\hline 68 & $6.837 \mathrm{de}$ & $11.633 \mathrm{c}$ & $1.207 \mathrm{~d}$ & $1.223 \mathrm{~d}$ & $1.334 \mathrm{de}$ & $1.474 d$ \\
\hline 137 & $13.236 b$ & $13.839 b$ & $2.267 \mathrm{a}$ & $2.223 \mathrm{a}$ & $5.497 \mathrm{a}$ & $4.960 \mathrm{~b}$ \\
\hline 205 & $5.862 \mathrm{e}$ & $6.751 \mathrm{de}$ & $1.481 \mathrm{c}$ & $1.574 b c$ & $4.406 \mathrm{c}$ & $5.721 \mathrm{a}$ \\
\hline
\end{tabular}

W64Ao2

$\begin{array}{rcccccc}0 & 11.436 \mathrm{e} & 15.890 \mathrm{c} & 3.474 \mathrm{a} & 2.548 \mathrm{~cd} & 513 \mathrm{c} & 496 \mathrm{c} \\ 68 & 13.527 \mathrm{~d} & 12.299 \mathrm{de} & 3.190 \mathrm{~b} & 2.421 \mathrm{~d} & 450 \mathrm{c} & 497 \mathrm{c} \\ 137 & 24.214 \mathrm{~b} & 26.527^{\mathrm{a}} & 2.792 \mathrm{c} & 3.181 \mathrm{~b} & 1.478 \mathrm{a} & 1.271 \mathrm{~b} \\ 205 & - & - & - & - & - & -\end{array}$

Os valores com a mesma letra, para cada variável e genótipo, não apresentam diferença significativa, pelo teste de Tukey ao nível de $5 \%$ de probabilidade.

de estresse já foi registrado anteriormente (Tattini et al., 1993; Willadino et al., 1996; Camara et al., 1998) e parece ter assegurado aos calos de W64Ao2 um melhor crescimento em relação aos calos de Arizona 8601 (Tabela 1).

Considerando-se os calos de ambos os genótipos quando cultivados em presença de Pro, observou-se um aumento na concentração de Put a partir da dose de $137 \mathrm{mM}$ de $\mathrm{NaCl}$ (Tabela 4). $\mathrm{O}$ acúmulo dessa diamina foi acompanhado pelo aumento na concentração de seu precursor, o aminoácido Arg (Tabela 2), o que sugere uma ativação da enzima ADC (arginina carboxilase) em condições de estresse, como constatado por outros autores (Smith, 1985; Flores, 1990). 
TABELA 4 - Concentração de poliaminas totais (PAs), putrescina, espermidina e espermina em calos de dois genótipos de milho, em função do tratamento com (c) e sem (s) prolina e dos níveis de $\mathrm{NaCl}$.

\begin{tabular}{|c|c|c|c|c|c|c|c|c|}
\hline \multirow{2}{*}{$\begin{array}{c}\mathrm{NaCl} \\
\mathrm{mM}\end{array}$} & \multicolumn{2}{|c|}{ Total de PAs } & \multicolumn{2}{|c|}{ Putrescina } & \multicolumn{2}{|c|}{ Espermidina } & \multicolumn{2}{|c|}{ Espermina } \\
\hline & s/Pro & c/Pro & s/Pro & c/Pro & s/Pro & c/Pro & s/Pro & c/Pro \\
\hline
\end{tabular}

Arizona 8601

$\begin{array}{rrrrrrrrr}0 & 1.161 \mathrm{c} & 896 \mathrm{e} & 393 \mathrm{~d} & 394 \mathrm{~d} & 202 \mathrm{c} & 224 \mathrm{c} & 566 \mathrm{~b} & 278 \mathrm{e} \\ 68 & 867 \mathrm{e} & 1.702 \mathrm{~b} & 370 \mathrm{de} & 363 \mathrm{de} & 205 \mathrm{c} & 676 \mathrm{a} & 292 \mathrm{e} & 663 \mathrm{a} \\ 137 & 660 \mathrm{f} & 1.014 \mathrm{~d} & 304 \mathrm{e} & 576 \mathrm{~b} & 94 \mathrm{e} & 148 \mathrm{~d} & 262 \mathrm{e} & 290 \mathrm{e} \\ 205 & 1.165 \mathrm{c} & 1.913 \mathrm{a} & 501 \mathrm{c} & 1.299 \mathrm{a} & 201 \mathrm{c} & 271 \mathrm{~b} & 463 \mathrm{c} & 343 \mathrm{~d}\end{array}$

W64Ao2

$\begin{array}{rrrrrrrrr}0 & 1.296 \mathrm{e} & 1.586 \mathrm{~d} & 560 \mathrm{f} & 930 \mathrm{~d} & 465 \mathrm{e} & 438 \mathrm{ef} & 271 \mathrm{f} & 218 \mathrm{~g} \\ 68 & 1.622 \mathrm{~d} & 1.023 \mathrm{f} & 794 \mathrm{e} & 376 \mathrm{~g} & 413 \mathrm{f} & 355 \mathrm{~g} & 415 \mathrm{c} & 292 \mathrm{e} \\ 137 & 2.565 \mathrm{~b} & 1.819 \mathrm{c} & 1.354 \mathrm{~b} & 1.013 \mathrm{c} & 853 \mathrm{a} & 628 \mathrm{c} & 358 \mathrm{~d} & 178 \mathrm{~h} \\ 205 & 1.937 \mathrm{c} & 2.732 \mathrm{a} & 882 \mathrm{~d} & 1.590 \mathrm{a} & 572 \mathrm{~d} & 688 \mathrm{~b} & 483 \mathrm{a} & 454 \mathrm{~b}\end{array}$

Os valores com a mesma letra, para cada variável e genótipo, não apresentam diferença significativa, pelo teste de Tukey ao nível de $5 \%$ de probabilidade.

Em síntese, os tratamentos com $\mathrm{NaCl}$ promoveram uma redução na taxa de crescimento dos calos. A adição de prolina ao meio de cultura favoreceu o crescimento dos calos no tratamento sem $\mathrm{NaCl}$ e reduziu o efeito prejudicial do mais alto nível de sal, em ambos genótipos. Os calos de
W64Ao2 mantiveram uma taxa de crescimento positiva na maioria dos tratamentos com $\mathrm{NaCl}$, possivelmente em função de um maior acúmulo de prolina, bem como pela manutenção do metabolismo das poliaminas em condições de estresse salino. 


\section{REFERÊNCIAS}

ALIA, P.; SARADHI, P. and MOHANTY, P. Proline in relation to free radical production in seedlings of Brassica juncea raised under sodium chloride stress. Plant and Soil, 155/156:497-500, 1993.

BASU, R. and GOSH, B. Polyamines in various rice genotypes with respect to sodium chloride salinity. Plant Physiology, 82:575-581, 1991.

BELLINGER, Y; BENSAOUD, A.and LARHER, F. Physiological significance of proline accumulation, a trait of use to breeding for stress tolerance. In: ACEVEDO, E.; CONESA, A.P. AND SRIVASTAVA, J.P. (Eds.) Physiology- breeding of winter cereals for stressed mediterranean environments. INRA, 449-458. 1991.

BERTELI, F.; CORRALES, E.; GUERRERO, C.; ARIZA, M.J.; PILEGO, F. and VALPUESTA, L. Salt stress increase ferredoxin-dependent glutamate synthase activity and protein level in the leaves of tomato. Physiologia Plantarum, 93:259-264, 1995.

BOURGEAIS-CHAILLOU, P. and GUERRIER, G. Salt response in Lycopersicun esculentum calli and whole plants. Journal of Plant Physiology, 140:495-501, 1992.

CACHORRO, P.; ORTIZ, A. and CERDA, A. Growth, water relations and solute composition of Phaseolus vulgaris under saline conditions. Plant Science, 95:23-29, 1993.

CAMARA, T.R.; WILLADINO, L.; TORNE, J.M.; SANTOS, M.A. Efeito da putrescina e do estresse salino em calos de milho. Revista Brasileira de Fisiologia Vegetal, 10:153-156, 1998.

CLAPAROLS, I.; SANTOS, M.A. and TORNE, J.M. Influence of some exogenous amino acids on the production of maize embriogenic callus and on endogenous amino contents. Plant Cell, Tissue and Organ Culture, 34:1-11, 1993.
CHU, C.C.; WANG, C.C.; SUN, C.S.; CHU, C.Y. AND BI, F.Y. Establishment of an efficient medium for anther culture of rice, through comparative experiments on the nitrogen sources. Science Sinica, 18:659-668, 1975.

DAS, N.; MISRA, M. and MISRA, A. Sodium chloride salt stress induced metabolic changes in callus cultures of Pear millet: free solute accumulation. Journal of Plant Phisiology, 137: 244-246, 1990.

DAY, A.D. Registration of Arizona 8601 maize germoplasm for saline environments. Crop Science, 27:1069, 1987.

DELAUNEY, A.J. and VERMA, D.P.S. Proline biosyntesis and osmoregulation in plants. The Plant Journal, 4:215-223, 1993.

EVANS, P. Somaclonal variatons: genetic basis and breeding applications. Theoretical and Applied Genetic, 77:889-464, 1989.

FEDINA, I.S.; TSONEV, T.D. and GULEVA, E.I. ABA as a modulator of the response of Pisum sativum to salt stress. Journal of Plant Physiology, 143:245-249, 1994.

FLORES, H.E. Polyamines and Plant Stress. In: FLORES, H.E. (Ed.) Stress Response in Plants: Adaptation and Acclimatation Mechanisms. Willey-Liss Inc., 1980. p.217239, 1990.

FLORES, H.E. and GALSTON, A.W. Polyamine and plant stress: activation of biosyntesis by osmotic shock.. Science, 217:1259-1260, 1982.

GALIBA, G.; KAUR-SAWHNEY, R. and GALSTON, A.W. Chromosomal localization of osmotic and salt stress-induced differential alterations in polyamine content in wheat. Plant Science, 92:203-211, 1993.

GARCIA, A.B.; ENGLER, J.A.; IYER, S.; GERAT, S.T.; MONTAGU, M.V. and CAPLAN, A.B. Effects of osmoprotectants upon $\mathrm{NaCl}$ stress in rice. Plant Physiology, 115:159-169, 1997. 
GREENWAY, H. and MUNS, R. Mechanism of salt tolerance in nonhalophytes. Annual Review of Plant Physiology, 31:149-190, 1980.

HANDA, S.; HANDA, A.K.; HASEGAWA, P.M. and BRESSAN, R. Proline accumulation and the adaptation of cultured plant cells to water stress. Plant Physiology, 80:938-945, 1986.

HASEGAWA, P.M.; BRESSAN, R.D. and HANDA, A.K. Cellular mechanism of salinity tolerance. HortScience, 21:1317-1324, 1986.

JAIN, S.; NAINAWATEE, H.S.; JAIN, R.K. and CHOWDHURY, J. B. Proline status of genetically stable salt-tolerant Brassica juncea somaclones and their parent cv. Prakash. Plant Cell Reports, 9:684-687, 1991.

KIRYAN, I.G. and SHEVYAKOVA, N.I. Proline accumulation pathway in $\mathrm{NaCl}$ resistant line of Nicotiana sylvestris cells. Fiziol Rast, 31:712720, 1984.

KUZNETSOV, V.V. and SHEVYAKOVA, N.I. Stress response of tobaco cells to high temperature and salinity. Proline accumulation and phosphorylation of polypeptides. Physiologia Plantarum, 100:320-326, 1997.

LOVATT, C.J. Stress alters ammonia and arginine metabolism. In: FLORES, H.E.; ARTECA, R.N. and SHANNON, J.C. (Eds.) Polyamines and ethylene biochemistry, physiology and interactions. American Society of Plant Physiologist, 1990. p.166-179.

PONS, A.; ESTEVES, T.; PONS, S. and ERITJA, R. Determinación del contenido de hidroxiprolina en el cuero. In: CONGRESO DE LA IUTCS,11, Barcelona,1991. Anales. Barcelona, 1991. p.24-31.

RABE, E and LOVATT, C. Increased arginine biosynthesis during phosphorus deficiency. Plant Physiology, 81:774-779, 1986.

ROOSENS, N.H.C.J.; TRAN, T.T.; HAYATI, M.I. and JACOBS, M. Isolation of the ornithine- $\alpha-$ aminotrasferase cDNA and effect of salt stress on ornithine expression in Arabdopsis thaliana. Plant Physiology, 117:263-271, 1998.

ROY, D.; BASU, N.; BHUNIA, A. and BANERJEE, S.K. Counteraction of exogenous L-proline with $\mathrm{NaCl}$ in salt-sensitive cultivar of rice. Biologia Plantarum, 35:69-72, 1993.

SHEVYAKOVA, N.I.; STROGONOV, B.P. and KIRYAN, G.I. Metabolism of polyamines in NaCl-resistant cell lines from Nicotiana sylvestris. Plant Growth Regulation, 3:365369, 1985.

SLOCUM, R.D. and WEINSTEIN, H. Stress induced putrescine accumulation as a mechanism of ammonia detoxification in cereal leaves. In: : FLORES, H.E.; ARTECA, R.N. and SHANNON, J.C. (Eds.) Polyamines and ethylene biochemistry, physiology and interactions. American Society of Plant Physiologist, 157-165, 1990.

SMITH, T.A. Polyamines. Annual Review of Plant Physiology, 36:117-143. 1985.

SONGSTAD, D.D.; DUNCAN, D.R. and WIDHOLM, J.M. Proline and polyamine involvement in chilling tolerance of maize suspension culture. Journal of Experimental Botany, 41:289-294, 1990.

STOREY, R.; GORHAM, J.; PITMAN, M.G.; HANSON, A.D. and CAGE, D. Response of Melantera biflora to salinity and water stress. Journal of Experimental Botany, 44:15511560. 1993.

TAL, M. Physiological genetics of salt resistance in higher plants. In: STAPLESS, R.C and TONNIESEN, H.E. (Eds.) Salinity tolerance in plants. Jonh Willey and Sons, 301-320, 1984.

TATTINI, B.Y.; HEIMLER, D.; TRAVERSI, M.L. and PIERONI, A. Polyamine analysis in salt stressed plants of olive. Journal of Horticutural Science, 68:613-617, 1993.

THOMPSON, J.F. Arginine synthesis, proline synthesis, and related process. In: STUMPF, 
E.E.; CONN, A. The Biochemistry of Plants. New York: Academic Press, 375-408, 1980.

TIBURCIO, A.F.; KAUR-SAWHNEY, R.; INGERSOLL, R.B. and GALSTON, A.W. Correlation between polyamines and pyrrolidine alkaloids in developing tobacco callus. Plant Physiology, 78:323-326, 1985.

van RENSBURG, L.; KRUGER, G.H.J. and KRUGER, $H$. Proline accumulation as drought-tolerance selection criterious: its relationship to membrane integrity and chloroplast ultraestructure in Nicotiana tabacum. Journal Plant Physiology, 141:188194, 1993.

VIÉGAS, R.A. and SILVEIRA, J.A.G. Ammonia assimilation and proline accumulation in young cashew plants during long term exposure to NaCl-salinity. Revista Brasileira de Fisiologia Vegetal, 11:153-159, 1999.
VIÉGAS, R.A.; MELO, A.R.B. and SILVEIRA, J.A.G. Nitrate reductase activity and proline accumulation in cashew in response to salt $(\mathrm{NaCl})$ shock. Revista Brasileira de Fisiologia Vegetal, 11:21-28, 1999.

VOETBERG, G.S. and SHARP, R.E. Growth of the maize primary root at low water potentials. III. Role of increased proline deposition in osmotic adjustment. Plant Physiology, 96:11251130, 1991.

WILLADINO, L.; CAMARA, T.; BOGET, N.; CLAPAROLS, I. and TORNE, J.M. Polyamine and free amino acid variations in $\mathrm{NaCl}$-treated embryogenic maize callus from sensitive and tolerant cultivars. Journal of Plant Physiology, 147:179-185, 1996. 